\title{
Motivation, Optimal Experience and Flow in First Year Computing Science
}

1st Author

4th Author 2nd Author

5th Author

\begin{abstract}
We examine the concept of motivation from the perspective of Self Determination Theory and give a brief overview of relevant results. We also consider the optimal state known as "Flow" and give an account of its conceptualisation in the theory due to Csikszentmihalyi. After discussion of ways in which these concepts can be measured, we describe a set of preliminary studies which looks at motivation and flow in the context of a first year computing class. We analyse student responses to enquiries about perceptions of motivation and flow experiences and look at links between them. We also discuss intrinsic motivation within the subject.
\end{abstract}

\section{Categories and Subject Descriptors}

K.3.2 [Computers and Education]: Computer and Information Science Education - computer science education, information systems information.

\section{General Terms}

Human Factors

\section{Keywords}

Motivation, self determination theory, optimal experience, flow,

\section{INTRODUCTION}

The factors that affect how an individual student engages with a course of study are often complex and multifaceted, dependent upon a range of cognitive, affective and social considerations [1]. However, there is good evidence that students are much more likely to persist in higher education if they are psychologically invested in the experience of learning than if not [2,3] This seems intuitively obvious, and providing opportunities for learning that are both academically meaningful and cognitively rewarding is a fundamental part of professional teaching activity. Motivation, thought of as the aspect of intentionality that focuses on direction and reasons to accomplish a task, is understood to play a foundational role in such concepts as self-efficacy $[4,5]$ and self-regulation [6]. Students themselves report that enhanced learning may follow from a variety of states characterised by high levels of engagement and increased motivation, but educational psychology research is particularly strong when considering those in which motivation is, to a greater extent, generated from within

Permission to make digital or hard copies of all or part of this work for personal or classroom use is granted without fee provided that copies are not made or distributed for profit or commercial advantage and that copies bear this notice and the full citation on the first page. To copy otherwise, or republish, to post on servers or to redistribute to lists, requires prior specific permission and/or a fee.

Conference'10, Month 1-2, 2010, City, State, Country.

Copyright 2010 ACM 1-58113-000-0/00/0010 ...\$15.00. the task itself and not forced by external constraints [7].

This result is clearly important from a pedagogical perspective and has implications for the way educators develop, deliver and assess learning activities. We would therefore like to know more about this in a computer science education context.

This paper draws on two main theories from educational psychology to investigate the experience of optimal states described by some students when learning aspects of computer science. Our aim is to try to situate the experience of a student who reports such a state within the broader theoretical context of those reported in other academic disciplines, and more widely, in other individual or social activities. To do this, we draw on the concepts and terminology of Self Determination Theory (SDT) [7, 8] to describe various levels of motivation experienced by a student when undertaking an activity. This approach proposes that the degree of motivation depends on the individual's "locus of control" [9] i.e. the extent to which that person believes that they can control the events affecting them. It is a well-established theory which evolved out of attempts to account for the effects of extrinsic rewards on intrinsic motivation, specifically the "Overjustification effect", i.e. the observation that, somewhat counter-intuitively, such rewards do not always motivate persistence in challenging tasks, and in some cases may serve to undermine it [10].

Since motivation is a psychological construct and therefore not directly observable, factors associated with engagement such as activation, persistence and intensity, are generally taken as proxies and assumed to correlate with it. An increase in the likelihood that a person will initiate an action, greater effort to persist in the face of challenges, and more intense activity in pursuit of goals all contribute to an operation definition of motivation. Given this, the analysis provided by self determination theory suggests that increases in these observable factors are generally correlated with increased levels of intrinsic motivation, i.e. when an individual finds a task rewarding for its own sake.

One such state is that of "optimal experience" or, to use the terminology of Csikszentmihalyi [11], "Flow". This phenomenon, in which the person feels simultaneously cognitively efficient, highly-motivated, and happy" [12] has been studied by researchers for over forty years and the concept has been found to be useful in a range of subject areas, especially in sport, music and art, where there is some degree of physical activity or performance. Flow states are characterised, within the execution of a task, by a merging of action and awareness, a centering of attention, and the loss of self-consciousness. Subjects speak of an immersive experience in which the person experiences a strong feeling of control and where requirements are clear and unambiguous. Such experiences are "autotelic" in nature, i.e. rewarding in themselves, without the need for external motivation. 
Within the broad field of computing, autotelic states such as flow have been studied in a range of contexts such as immersive gaming [13, 14] game-based learning [15] instructional design [16] Information Systems adoption [17] and HCI [18, 19, 20].

We believe that the concepts of flow, and autotelic behaviour in general, are becoming important features in a range of CS education contexts. These range from the programmer who becomes absorbed in the coding exercise to the student working on an open-ended project. The purpose of this paper is twofold. Firstly we seek to give a general and accessible account of some of the basic results associated with self determination theory and flow theory and secondly report on an initial study into the concepts in the context of first year computing science students. We analyse student responses to enquiries about perceptions of flow experiences and look at links between these reports and intrinsic motivation within the subject. We also investigate links with student perceptions of confidence and self-identity.

\section{BACKGROUND}

From the standpoint of practical pedagogy, understanding the basis for student motivation, and its expression in course engagement, is a key task that impacts critically on most aspects of teaching and learning. In what follows, we use Self Determination Theory to describe motivational states, while eventually focussing on Flow-states. We therefore give a brief, general account of both of these theories before looking at how these concepts are measured.

\subsection{Motivation and Self Determination Theory}

Early theories of motivation focused on a classification of motives in terms of needs and how these are fulfilled. So, for example, the theory proposed by Maslow [21] as well as later developments such as such as ERG theory [22] sought to describe motivation in terms of a hierarchy of needs, with the satisfaction of its lower levels (physiological, safety) being a prerequisite for engagement with the higher levels, culminating in the need for selfactualisation and self-transcendence.

While these general theories have proved useful in some fields such as sociology, they generally treat motivation as a unitary concept and this does not conform to experience within an educational context. One influential psychological theory that does attempt to consider different types of motivation is Self Determination Theory $[8,23]$. The theory attempts to give an account of the concept of motivation from both a social and a cognitive perspective using the degree of self-determination to characterise it along a continuum from least to most selfdetermined. It posits a broad distinction between intrinsic and extrinsic forms: the latter referring to initiation of activities in order to fulfil some external goal whereas the former is characterised by engagement in tasks for their own sake, regardless of any external reward structure.

Deci and Ryan proposed a motivation continuum based upon increasing levels of self-determination. At the lowest end is what they term "Amotivation", an absence of motivation characterised by a feeling of lack of control over actions and an absence of value derived from completion of tasks. This is followed by an intermediate level of extrinsic motivation, which itself can be differentiated into a number of sub-categories. At the lowest end of this intermediate scale is external regulation, where motivation is caused solely by external rewards and punishments. Above this is introjection in which individuals begin to internalise the reasons for their own behaviour and impose their own reward structures in terms of what they perceive they ought to do. Identification takes place when the individual identifies with the reason for behaving in a certain way and moves from normative to volitional justification. Finally, integrated regulation is said to occur when the extrinsic motivation is fully assimilated and accepted into the sense of self. The distinction between the first two extrinsic categories and the latter two was also stated using the terms nonself-determined extrinsic motivation for behaviour completely controlled by external factors contrasting with self-determined extrinsic motivation which occurs when the individual engages in an activity because of a personal choice and an attribution of socially determined values to the task.

\begin{tabular}{|c|c|c|}
\hline $\begin{array}{l}\text { Low self-determination } \\
\text { Low autonomy } \\
\text { Low sense of control }\end{array}$ & \multicolumn{2}{|c|}{$\begin{array}{r}\text { High self-determination } \\
\text { High autonomy } \\
\text { High sense of control }\end{array}$} \\
\hline \begin{tabular}{cl} 
Amotivation | & \multicolumn{2}{c}{ Extrinsic } \\
& | \\
I & External \\
& regulation \\
& Introjection
\end{tabular} & $\begin{array}{l}\text { Motivation } \\
\text { dentification } \\
\text { Integrated } \\
\text { regulation }\end{array}$ & $\begin{array}{l}\text { Intrinsic } \\
\text { Motivation }\end{array}$ \\
\hline
\end{tabular}

Figure 1. The Motivation Continuum (adapted from Deci \& Ryan, [23])

The highest form of self-determination occurs with intrinsic motivation where participation in some task is done for its own sake, because of personal interest or the satisfaction derived from the experience. Later development within the framework of SDT investigated the effect of contextual factors on intrinsic motivation and proposed a direct correlation between high intrinsic motivation and features that promote feelings of personal autonomy and competence. Personal growth and psychological well-being are enhanced when individuals try to gain as much autonomy over their own behaviour as possible and this occurs through the development of competence in both actions and the decision-making processes that lead to them [23]. Since highquality learning and creativity are often the result of the more selfdetermined form of motivation, a pedagogical priority would be to provide an environment that promotes this. Both intrinsic and the self-determined forms of extrinsic motivation have been found to be associated with positive educational outcomes such as greater engagement in learning [24], better performance [25], and greater psychological well-being [26].

The dual need for autonomy and competence is accompanied by a further motivational factor, that of the desire for relatedness. This is a contextual factor in which individuals seek to establish a psychological connection with others and is especially important in the setting of social interactions, such as those that occur in learning environments. SDT proposes that people have an innate tendency to internalise new knowledge and practices that are acquired through socialisation, and that satisfaction of the need for relatedness facilitates this process of internalisation. In particular, external motivators derived from the values and practices of other people with whom an individual feels (or desires to feel) some kind of connection, are accepted as his or her own and transformed into intrinsic motives. This would, for example, be important in an educational context where academic maturation is seen as a process by which the learner is inducted into a 
community of practice, taking on the norms and values of that community. In the context of secondary school, such relatedness has been shown to be associated with student perceptions of value and respect from teachers and parents and this fosters intrinsic motivation [27].

SDT therefore maintains that, when students' basic psychological needs for autonomy, competence, and relatedness are supported in the classroom, they are more likely to internalize their motivation to learn and to be more autonomously engaged in their studies.

Later elaboration of the theory [28] proposed a multidimensional picture of motivation and suggested that different motivational impulses needed to be incorporated in any comprehensive account of the concept. In sport science research, for example, more autonomous motivational factors such as self-determined extrinsic motivation have been linked to enhanced performance and more effective coping strategies in the face of set-backs [29], greater levels of persistence and higher levels of time investment in activities [30].

\subsection{The Concept of Flow}

While Deci, Ryan and their co-workers were investigating these concepts of motivation in the context of Self Determination Theory, another aspect of intrinsic motivation was developed by Csikszentmihalyi, the concept and theory of "Flow". Csikszentmihalyi [11] defined flow as "the holistic sensation that people feel when they act with total involvement". Following interviews with individuals pursuing a variety of different activities - artists, dancers, chess players, rock-climbers, surgeons - Csikszentmihalyi noticed a common element in their description of feelings of optimal performance: expressions such as "being in the midst of flow" or "flowing from one moment to the next" were often used to describe such experiences

Flow states are states of "optimal experience" in the sense that the person involved in the activity feels "simultaneously cognitively efficient, motivated, and happy" [31, 32]. Moreover, Csikszentmihalyi and others found that these states correlated with enhanced performance in a variety of creative activities, sporting activities, as well as learning [33, 34]. The enjoyable nature of flow promotes learning and engagement with more complicated activities since, to maintain the state, the subject of the experience has to maintain the balance between challenge and skills resulting in a synchronized increase in task difficulty as proficiency develops. It was this unfolding virtuous circle of selfactualisation in which an individual continually seeks out new tasks by setting, and ultimately surpassing, increasingly challenging problems, that Csikszentmihalyi saw as the key to a rewarding and productive life for an individual [35] and for also for a culture to flourish [36].

The conditions of flow include a sense that one is engaging in challenges at a level which is appropriate to one's capacities with having clear, proximal goals with immediate, accessible feedback about progress that is being made.

Csikszentmihalyi early work on flow describes six elements that characterise the state. The first is a "merging of action and awareness" in which a person is aware of the actions being performed but not of the awareness itself. There is a focusing or centering of attention on the specific details of the problem at hand. There is a loss of self-consciousness in which individual considerations become irrelevant to the task. This is accompanied by feeling of control or mastery over the performance of the task together with clear, unambiguous knowledge of the course of action clarified by immediate and plain feedback. Objectives are perceived as logically connected with a clear order of operation and reaction to subtasks is automatic. Finally, the state has an "autotelic" nature, that is, there is no need for external goals or rewards as the experience of participation is its own reward, i.e. it is intrinsically motivated.

In subsequent elaboration of the earlier theory, Csikszentmihalyi [35] and others also suggested that further characteristics of flow included a sense of "the distortion of temporal experience of time", i.e. that time seemed to go faster when the individual engaged in the activity in the flow-like state. In addition he looked at precursors or conditions that are required to exist before a flowlike state develops. The most important of these is that a person should have a feeling of control over the process with a balance between the challenge involved and the skills required to complete a task.

The challenge-skill requirement has led a number of researchers to try to develop a model of flow based on these components. One developed by Csikszentmihalyi and LeFevre [31] is the Quadrant model.

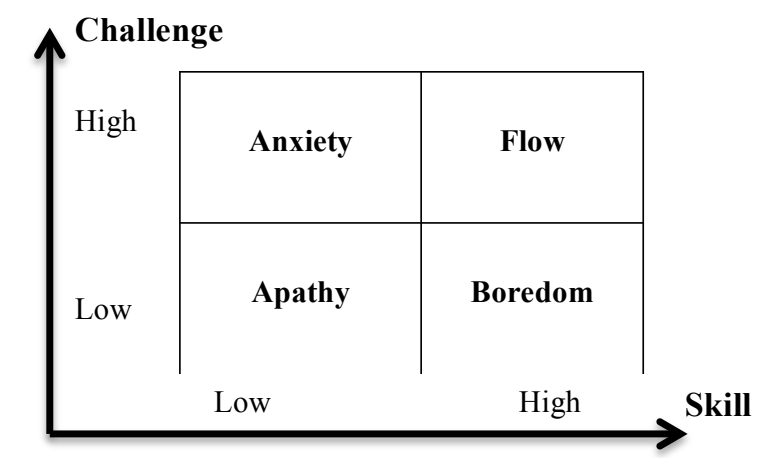

Figure 2. The quadrant model of the flow state (Adapted from Csikszentmihalyi and LeFevre 1989)

An expanded version, the Octal, or Experience Fluctuation model, was developed in Massimini et al. [36]. This kept the twocomponent axes of the model but included a finer grain classification of sectors in the diagram. It retained the apathy-flow characterisation of the low-to-high diagonal but included more nuanced states around these, corresponding to medium-level values of challenge and skill, e.g. "control" (high skill/medium challenge) and "worry" (low skill/medium challenge).

Whatever the precise details of the model, there seems to be agreement in the literature that flow is associated with high skill/high challenge situations and that the cognitive and affective reward experienced by the individual is such that they are more likely to engage in the activity again. This has implications for learning akin to the previous discussion about optimal states in the context of intrinsic motivation.

\section{METHOD}

We wished to investigate the concepts such intrinsic motivation and flow in the context of first year computing students. The question we wished to answer was whether the states described by students were similar to those found elsewhere in the literature and whether they exhibited the same structure as those found in flow questionnaires in other situations. Previous discussion with the individual members of the student cohort had attested to a general feeling that many of them had had prior experience of optimal states, which they themselves interpreted as flow, 
although not necessarily in a computing education context. Many of these had been in the more reported setting of computer gaming, musical or other artistic performance, or in sport. We wished to investigate whether they perceived being in such states in the context of their university computing education. In this initial study, we were not trying to check the veracity of the claims themselves, but simply trying to compare the characteristics of the states reported with those described elsewhere in the literature. Consequently, although we collected data from a general population of first year computing students, the data analysis was restricted those students who reported that they did have some flow-like experience. This reduced the sample size somewhat but we still feel the quantitative results obtained are useful for this initial study especially when taken in conjunction with more qualitative text-based data that was subsequently sought from individual students.

\subsection{The Participants}

Our study used data obtained from a group of forty first-year undergraduate students in Institution A. The students in the investigation were aged between 17 and 30 with the majority having entered university directly from secondary school. They were registered on four degrees within the School: the largest group was studying $\mathrm{X}$, with the remainder studying $\mathrm{Y}, \mathrm{Z}$ and $\mathrm{W}$. The first three of these courses had identical course units in the first year while the $\mathrm{W}$ students took the same subjects but with a programming course unit more tailored to $\mathrm{W}$ things. The students had completed the first semester course when the questionnaires were distributed. Results from 64 students were collected, of which 40 completed the two questionnaires and reported some experience of a state similar to flow.

\subsection{The Questionnaires}

The students were asked to complete two questionnaires. The first was a 24-item motivation survey based on a subject-specific version of the Sports Motivation Scale-6 (SMS-6) [37], which was itself an adaptation of the academic motivation scale (AMS) [38]. It was decided to use the SMS-6 rather than the original AMS because the latter did not address specifically the most autonomous form of extrinsic motivation, integrated regulation. Since our main interests were precisely in the more self determined forms of motivation, this was not appropriate for us. In addition, SMS-6 had previously reported high levels of convergent validity with measures of flow. The use of the SMS-6 outside its original domain of application (sports science) required some slight alteration of the text to refer to computing rather than sporting activity but this was fairly straightforward as the original had been developed from the more general AMS and many of the questions reflected this, e.g. in most case it was possible to substitute "computing activity" for "sporting activity".

The questionnaire itself comprised twenty-four statements and was constructed to track 6 factors associated with types of motivation in self determination theory, namely amotivation, the four subcomponents of extrinsic motivation, and intrinsic motivation. The data collected consisted of responses to the question "Why are you studying computing?" These responses comprised a set of statements, agreement with which was rated on on a seven-point Likert scale from 1 ("Does not correspond at all") to 7 ("Corresponds exactly"). The statements included in the questionnaire were divided into groups corresponding to the motivational typology described by SDT. So, for example, those corresponding to amotivation (lack of perceived control) include "I have the impression of being incapable of succeeding in this subject", and "I don't seem to be enjoying computing as much as I did" while those for identified regulation (where the person starts to internalise the externally motivated reasons for doing something) include "'Because it is a good way for me to learn lots of things that would be useful to me in other areas of my life" and "Because it is one of the best ways to maintain good relationships with my friends". Also, intrinsic motivation is addressed with statements such as "For the excitement I feel when I am really involved in the activity" and "For the satisfaction I experience when perfecting my abilities".

The second questionnaire was the Flow-State Scale (FSS) [39]. This is a well-established survey instrument that attempts to measure flow in the context of sport or physical exercise. As with the SMS-6, this FSS required some textual change in order to refer to a computing context rather than a sporting one but this was straightforward to accomplish. The questionnaire is made up of 36 items divided into 9 components that track elements described by flow research. These consist of autotelic experience, clarity of goals, the balance between challenge and skill, concentration on task, feeling of control, unambiguous feedback, the merging of action and awareness, the loss of selfconsciousness and the transformation of time. Students were asked to recall one specific instance that occurred while participating in the programming module that they believed constituted an optimal experience in the sense described previously. Response was then given to the items in the flow questionnaire using a 5-point Likert scale where 1 indicates "Strongly disagree" and 5 indicates "Strongly agree". For example, one statement tracking autotelic experience are "the experience was really rewarding", one for challenge-skill balance is "I was challenged but believed my skills would allow me to meet the challenge", while an example of a statement tracking concentration on task is "My attention was focussed entirely on what I was doing".

\section{RESULTS AND DISCUSSION}

Factor analysis of the questionnaires yielded results that were generally in line with those reported in other research. For the Motivation questionnaire, both the scree plot of the principal components and Kaiser's criterion (eigenvalue $>1$ ) indicate a 6factor model in agreement with the claims for SMS-6. Examination of the model structure with 5 and 7 factors did not appear to facilitate greater identification of the factors themselves.

Internal consistency of the responses to the components within the questionnaire was acceptable with Cronbach alpha scores in the range $0.68-0.87$. Given the rotated factor loadings and the questionnaire rubric, attempts to identify the factors suggested strong evidence for the components of Introjection and Amotivation. There was less definitive although still reasonable evidence for Integrated Regulation and Intrinsic Motivation and some for External Regulation. The last factor was difficult to identify but appears to be a mixture of Identified Regulation with Integrated Regulation, and therefore would represent the initial stages of self-determined extrinsic regulation according to self determination theory.

Turning to the Flow State Scale questionnaire, we again see broad agreement with results from other fields, although there is more variation with this questionnaire. Factor analysis yields a scree plot of the principal components that has one large eigenvalue with a number of smaller ones. Kaiser's criterion gives 9 factors (although this is not necessarily obvious from the plot itself which has inflection points at eigenvalues $1,4,8$ followed by a smooth 
decreasing plot). This suggests that identification of the factors is difficult and this is indeed what is found.

The first factor is a mixture of components associated with the "Challenge-Skill Balance" and "Merging of Action and Awareness". The second factor appears to be associated with "Clarity of Goals" and "Unambiguous Feedback" while the third factor is "Concentration on the Task at Hand". The fourth factor has a mixture of responses about "Transformation of Time" and "Loss of self-Consciousness". The identity of factors after this becomes difficult as the loadings become smaller and no set of value tends to dominate. It should be noted however that a similar factor analysis carried out with 8 factors also gave rise to identification of these factor groupings although not in the same order. It is therefore reasonable to infer that the states described by students could be characterised, at least in a first approximation, by elements such as the balance between challenge and skills and a merging of action and awareness, clear objectives provided by unambiguous feedback they were receiving from the task, a high degree of concentration on the narrow remit of the task in hand, and some kind of absorption factor associated with not being conscious that time was passing more quickly.

While not reproducing the full set of factors associated with the Flow-State Scale, the results do provide some insight into the experiences reported by students. Furthermore, although, due to space limitations, we do not report here on the information gained from the more qualitative data obtained from student, this tends to corroborate the perception of flow states in terms of the variables described above.

The validity of the results reported here can of course be challenged on a number of grounds. The sample size $(\mathrm{N}=40)$ is small. Nevertheless, the results are broadly comparable, at least qualitatively, with those found in in more extensive studies on the subject in areas such as sports science. It is also recognised that this initial study says nothing about those students who claim not to have such optimal experiences at any point in their educational journey through computing.

\section{CONCLUSION}

While the results discussed above are only an initial foray into the study of optimal experience in computer science education, we do believe that the subject is deserving of further, more systematic investigation. The link between engagement and intrinsic types of motivation appears to be both intuitively reasonable and fairly well documented in the educational psychology research literature. Furthermore students do appear to report these states in other areas such as leisure activities, performance arts and sport.

Of course, the fact that a student reports some degree of absorption and loss of self-consciousness in one particular activity, e.g. playing an immersive video game, does not necessarily imply that this state is the same as one that occurs in a learning context, say, a particularly engaging coding exercise. Nor, by itself, does it signify the existence of an optimal state such as flow. It may also be that, even if such states do exist in a noneducational situation, their benefits are not transferable to a pedagogical context.

Nevertheless, even if they only serve only to motivate students to engage with an activity in order to recapture affective aspects of the optimal experience, this would enhance engagement and so there may well be merit in further investigation. The idea that a significant aim of education is to promote intrinsic, or at least self- determined extrinsic, motivation for activities is an attractive one, and something that would be supported in the context of lifelong learning. While computer science education is necessarily concerned with the development of technical skills and professional competencies that allow students to gain employment in the computing industry, we would also wish to facilitate and validate the optimal experiences that some students have while studying the subject. States of intrinsic motivation, like flow, which appear to set up a virtuous circle in which students themselves desire to perform in challenging situations that pushes the limits of their skills and prompts them to develop greater expertise, also appears to be relevant to questions about student identity. Students who report flow-like experiences are more likely to identify as a member of the practising community and some of the questions on the Flow-State Scale questionnaire concern identity in terms of the subject, e.g. "I do this because I am a computer scientist" but also learning identity in the sense of Dweck's Self theory [40]. We therefore believe that the concept will be of use in the future and that further work in this area is warranted.

\section{REFERENCES}

[1] Trowler, Vicki. "Student engagement literature review." York: Higher Education Academy (2010).

[2] Moxley, David, Anwar Najor-Durack, and Cecille Dumbrigue. "Keeping Students in Higher Education: Successful Practices \& Strategies for Retention." (2001).

[3] Shaun, R., and Stephen John. "Student Engagement in Higher Education: Theoretical Perspectives and Practical Approaches for Diverse Populations." (2009).

[4] Bandura, Albert. "Self-efficacy: toward a unifying theory of behavioral change." Psychological review 84.2 (1977): 191.

[5] Zimmerman, Barry J. "Self-efficacy: An essential motive to learn." Contemporary educational psychology 25.1 (2000): 82-91.

[6] Schunk, Dale H., and Barry J. Zimmerman. "Self-regulation and learning." Handbook of Psychology (2003).

[7] Deci, E., \& Ryan, R. (2008). Self-determination Theory: A Macrotheory Of Human Motivation, Development, And Health. Canadian Psychology/Psychologie Canadienne, 49(3), 182-185.

[8] Deci, E. L., \& Ryan, R. M. (1985). Intrinsic motivation and self-determination in human behavior. New York: Plenum.

[9] Rotter JB (1954) Social Learning and Clinical Psychology. Englewood Cliffs, NJ: Prentice Hall.

[10] Deci, E. (1971). Effects Of Externally Mediated Rewards On Intrinsic Motivation. Journal of Personality and Social Psychology,18(1), 105-115.

[11] Csikszentmihalyi, M., 1975. Play and intrinsic rewards. Journal of humanistic psychology, Vol 15(3), 1975, 41-63.

[12] Csikszentmihalyi, M. (1990). Flow: The psychology of optimal experience. New York: Harper \& Row.

[13] Faiola, A. and Voiskounsky, A.E., 2007. Flow experience of MUD players: Investigating multi-user dimension gamers from the USA. In Online Communities and Social Computing (pp. 324-333). Springer Berlin Heidelberg

[14] Penelope Sweetser and Peta Wyeth. Gameflow: A model for evaluating player enjoyment in games. Comput. Entertain., $3(3): 3-3,2005$. 
[15] Faiola, A., Newlon, C., Pfaff, M. and Smyslova, O., 2013. Correlating the effects of flow and telepresence in virtual worlds: Enhancing our understanding of user behavior in game-based learning. Computers in Human Behavior, 29(3), pp.1113-1121.

[16] Chan, T.S. and Ahern, T.C., 1999. Targeting motivationadapting flow theory to instructional design. Journal of Educational computing research, 21(2), pp.151-163.

[17] Lowry, P.B., Gaskin, J., Twyman, N., Hammer, B. and Roberts, T., 2012. Taking 'Fun and Games' Seriously: Proposing the Hedonic-Motivation System Adoption Model (HMSAM). Journal of the Association for Information Systems, 14(11), pp.617-671.

[18] Ghani, J.A. and Deshpande, S.P., 1994. Task characteristics and the experience of optimal flow in human-computer interaction. The Journal of psychology, 128(4), pp.381-391.

[19] Finneran, C. and Zhang, P., 2002. The challenges of studying flow within a computer-mediated environment. AMCIS 2002 Proceedings, p.146.

[20] Saket, Bahador, Carlos Scheidegger, and Stephen Kobourov. "Towards Understanding Enjoyment and Flow in Information Visualization." arXiv preprint arXiv:1503.00582 (2015).

[21] Maslow, Abraham Harold. "A theory of human motivation." Psychological review 50.4 (1943): 370.

[22] Alderfer, Clayton P. "An empirical test of a new theory of human needs." Organizational behavior and human performance 4.2 (1969): 142-175.

[23] Ryan, R., \& Deci, E. (2000). Intrinsic and extrinsic motivations: Classic definitions and new directions. Contemporary educational psychology, 25(1), 54-67.

[24] Connell, James P., and James G. Wellborn. "Competence, autonomy, and relatedness: A motivational analysis of selfsystem processes." (1991).

[25] Miserandino, Marianne. "Children who do well in school: Individual differences in perceived competence and autonomy in above-average children." Journal of Educational Psychology 88.2 (1996): 203.

[26] Sheldon, Kennon M., and Tim Kasser. "Coherence and congruence: two aspects of personality integration." Journal of personality and social psychology 68.3 (1995): 531.

[27] Grolnick, W.S. and Ryan, R.M., 1989. Parent styles associated with children's self-regulation and competence in school. Journal of educational psychology, 81(2), p.143.

[28] Vallerand, Robert J. "Toward a hierarchical model of intrinsic and extrinsic motivation". In Zanna, Mark P. (Ed), (1997). Advances in experimental social psychology, Vol.
29. , (pp. 271-360). San Diego, CA, US: Academic Press, ix, $366 \mathrm{pp}$

[29] Amiot, C.E., Gaudreau, P. and Blanchard, C.M., 2004. Selfdetermination, coping, and goal attainment in sport. Journal of Sport and Exercise Psychology, 26(3), p.396.

[30] Pelletier, L.G., Fortier, M.S., Vallerand, R.J. and Briere, N.M., 2001. Associations among perceived autonomy support, forms of self-regulation, and persistence: A prospective study. Motivation and emotion, 25(4), pp.279306.

[31] Csikszentmihalyi, M. and LeFevre, J., 1989. Optimal experience in work and leisure. Journal of personality and social psychology, 56(5), p.815.

[32] Moneta, G.B. and Csikszentmihalyi, M., 1996. The effect of perceived challenges and skills on the quality of subjective experience. Journal of personality, 64(2), pp.275-310.

[33] Rathunde, K. and Csikszentmihalyi, M., 2005. Middle school students' motivation and quality of experience: A comparison of Montessori and traditional school environments. American Journal of Education, 111(3), pp.341-371.

[34] Engeser, S. and Rheinberg, F., 2008. Flow, performance and moderators of challenge-skill balance. Motivation and Emotion, 32(3), pp.158-172.

[35] Csikszentmihalyi, M., 1996. The creative personality. Psychology Today, 29(4), pp.36-40.

[36] Massimini, F., Csikszentmihalyi, M. and Fave, A.D., 1988. Flow and biocultural evolution. In Csikszentmihalyi, Mihaly (Ed); Csikszentmihalyi, Isabella Selega (Ed), (1988). Optimal experience: Psychological studies of flow in consciousness. , (pp. 60-81). New York, NY, US: Cambridge University Press, xiv, 416 pp.

[37] Mallett, C., Kawabata, M., Newcombe, P., Otero-Forero, A. and Jackson, S., 2007. Sport motivation scale-6 (SMS-6): A revised six-factor sport motivation scale. Psychology of Sport and Exercise, 8(5), pp.600-614.

[38] Vallerand, R. J., Pelletier, L. G., Blais, M. R., Briere, N. M., Senecal, C., \& Vallieres, E. F. (1992). The academic motivation scale: A measure of intrinsic, extrinsic, and amotivation in education. Education and PsychologicalMeasurement, 52, 1003-1017.

[39] Jackson, S.A. and Marsh, H.W., 1996. Development and validation of a scale to measure optimal experience: The Flow State Scale. Journal of sport and exercise psychology, 18, pp.17-35.

[40] Dweck, C. S. 2000. Self-theories: Their role in motivation, personality, and development. Psychology Press. 